\title{
Reconstruction and Identification of Hadronically Decaying Tau Leptons at ATLAS
}

\author{
David Jennens* \\ The University of Melbourne \\ on behalf of the ATLAS Collaboration \\ E-mail: david.thomas.jennens@cern.ch
}

\begin{abstract}
Tau leptons play an important role in the physics program at the LHC. They are used not only in searches for new phenomena like the Higgs boson or Supersymmetry and electroweak measurements, but also in detector related studies like the determination of the missing transverse momentum performance. Variables describing the properties of calorimeter energy deposits and track reconstruction within tau candidates are combined in multi-variate discriminants to achieve high rejection against backgrounds. The identification efficiencies are measured using $W \rightarrow \tau v$ and $Z \rightarrow \tau \tau$ events, and compared with the prediction of the Monte Carlo simulation. The energy scale uncertainties for tau leptons are determined by investigating single hadron calorimeter response, as well as kinematic distributions in $Z \rightarrow \tau \tau$ events.
\end{abstract}

The XIth International Conference on Heavy Quarks and Leptons,

June 11-15, 2012

Prague, Czech Republic

${ }^{*}$ Speaker. 


\section{Introduction}

Tau leptons play a central role in the LHC physics program, in particular as a signature for the Higgs boson and Supersymmetry searches. Tau leptons are used in Standard Model electroweak measurements, as well as detector related studies, like the determination of the missing transverse momentum performance. Tau leptons have a short lifetime $(c \tau=87 \mu \mathrm{m})$ and so predominantly decay inside the ATLAS beam pipe. Of these decays $64.7 \%$ are hadronic, consisting primarily of one or three charged pions, neutral pions and a neutrino. Depending on the number of charged pions such decays are categorised as either "1-prong" or "3-prong". The greatest challenge is the identification of hadronic tau decays against QCD jets, which appear similar and have a high production cross-section. In addition, electrons can be mis-identified as 1-prong tau decays. Separate techniques are developed for discrimination against electrons. As hadronic tau decays consist of a specific mix of charged and neutral pions, the energy scale of hadronic tau candidates (TES) is derived independently of the jet energy scale. The systematic error associated to the TES is determined using a mix of data driven and simulation based methods.

\section{Reconstruction}

Tau leptons which decay to hadrons are built from calorimeter jets reconstructed with the anti- $k_{t}$ algorithm [1] using a distance parameter $R=0.4$, which have a transverse momentum of at least $10 \mathrm{GeV}$ and are with $|\eta|<2.5$. The reconstruction algorithm attempts to reconstruct the four-momentum of all decay products except the neutrino. Tracks and clusters within a cone of $\Delta R^{1}=0.2$ from the tau-jet axis are associated to the tau candidate provided they pass certain quality requirements. Tracks and clusters in a wider cone of $\Delta R=0.4$ are also used to derive inputs for identification algorithms.

\section{Identification}

As tau reconstruction provides no discrimination between quark or gluon initiated jets and hadronic decays of tau leptons, identification is performed using distinct identification algorithms [2]. These algorithms take as inputs various quantities built from the calorimeters or the inner detector, most notably the shower width in the calorimeter (Figure 1) and the number of tracks in the annulus $0.2<\Delta R<0.4$ from the tau-jet axis (Figure 2).

Three independent discriminants are used to identify tau leptons decaying to hadrons. A selection of rectangular cuts, a projective log-likelihood score and a score derived from boosted decision trees (BDT). Each of the discriminants is trained to three working points in signal efficiency of approximately $60 \%$ (Loose), 45\% (Medium) and 30\% (Tight). The discriminants use different input variables and are optimised separately for 1-prong and 3-prong taus. For the training the algorithms use samples of simulated $W \rightarrow \tau \nu$ and $Z \rightarrow \tau \tau$ events for the signal and dijet events selected from 2011 data. Background rejection power is shown as a function of signal efficiency for each discriminant for 1-prong taus (Figure 3) and 3-prong taus (Figure 4) in the region $20 \mathrm{GeV}<p_{T}<40 \mathrm{GeV}$.

\footnotetext{
${ }^{1} \Delta R=\sqrt{(\Delta \eta)^{2}+(\Delta \phi)^{2}}$
} 


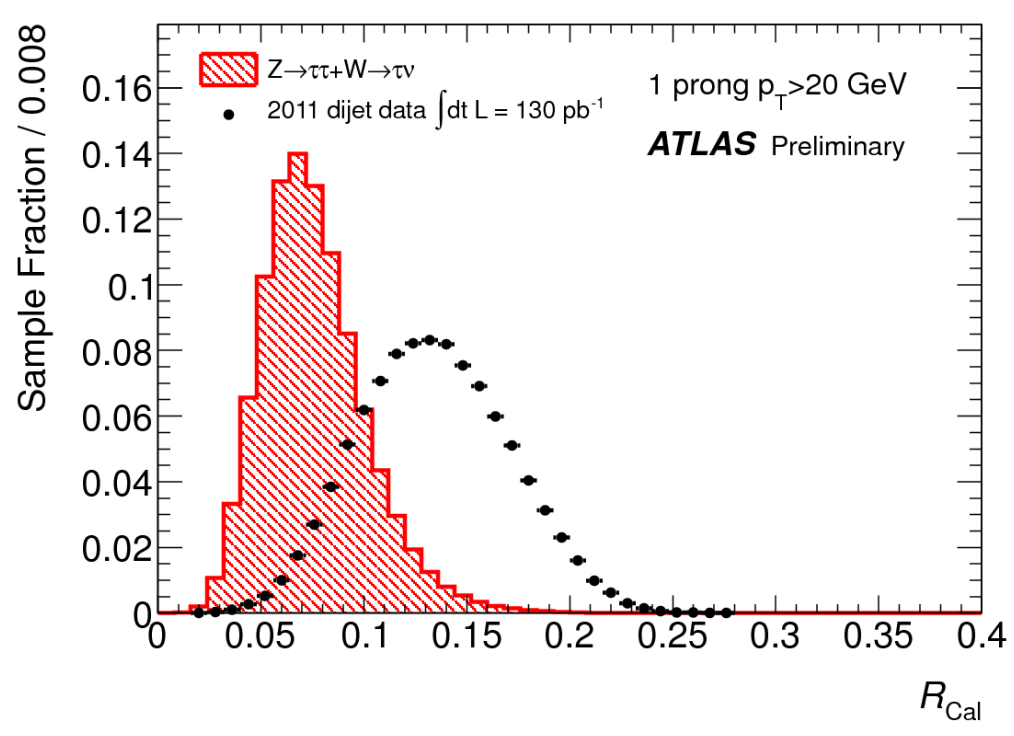

Figure 1: Shower width in the electromagnetic and hadronic calorimeter weighted by the transverse energy of each calorimeter part for MC simulated tau samples (red) and dijet sample selected from 2011 data (black). Both distributions are normalised to unity [2].

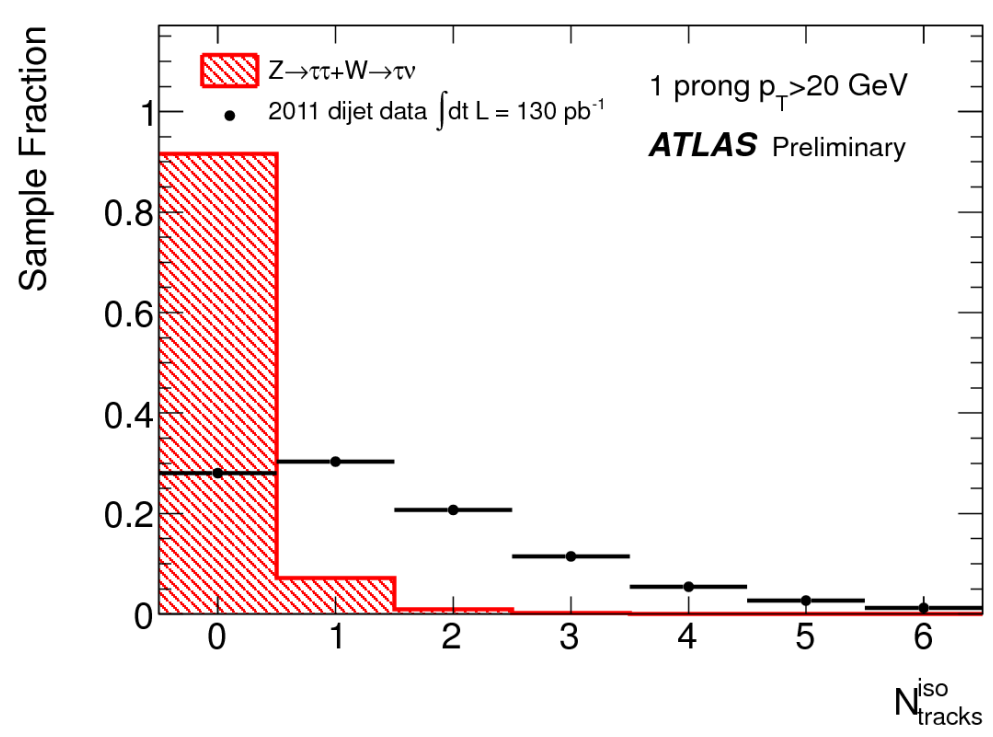

Figure 2: Number of tracks in the annulus $0.2<\Delta R<0.4$ from the tau-jet axis for MC simulated tau samples (red) and QCD dijet sample selected from 2011 data (black). Both distributions are normalised to unity [2]. 


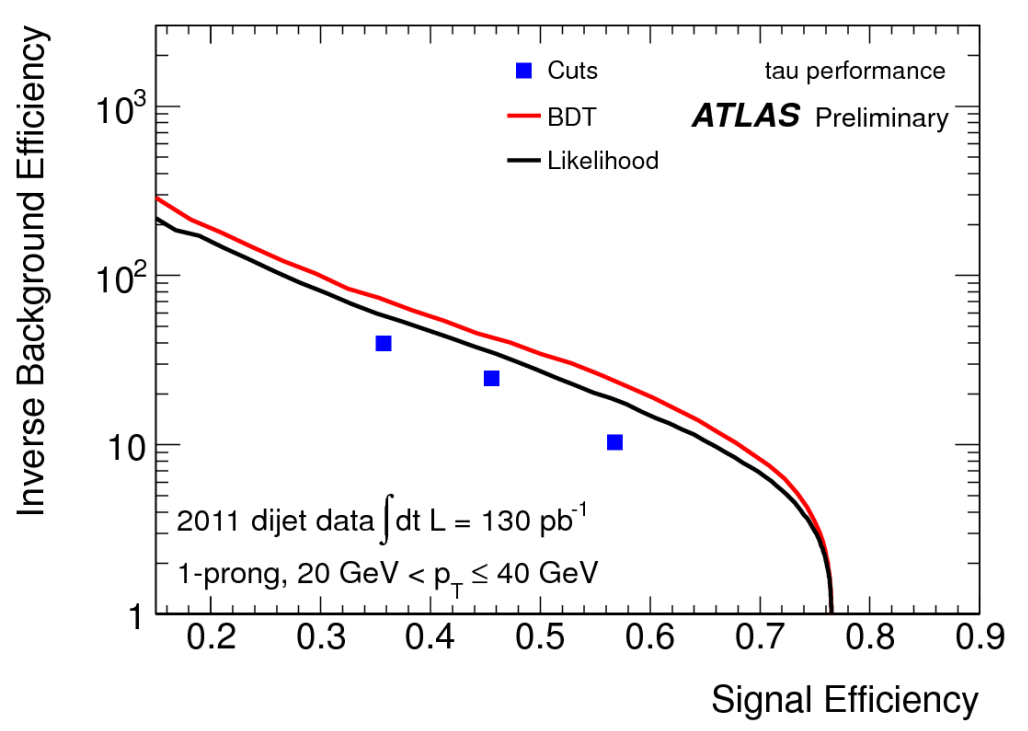

Figure 3: Inverse background efficiency as a function of signal efficiency for each jet discriminant for 1-prong taus in the region $20 \mathrm{GeV}<p_{T}<40 \mathrm{GeV}$ [2].

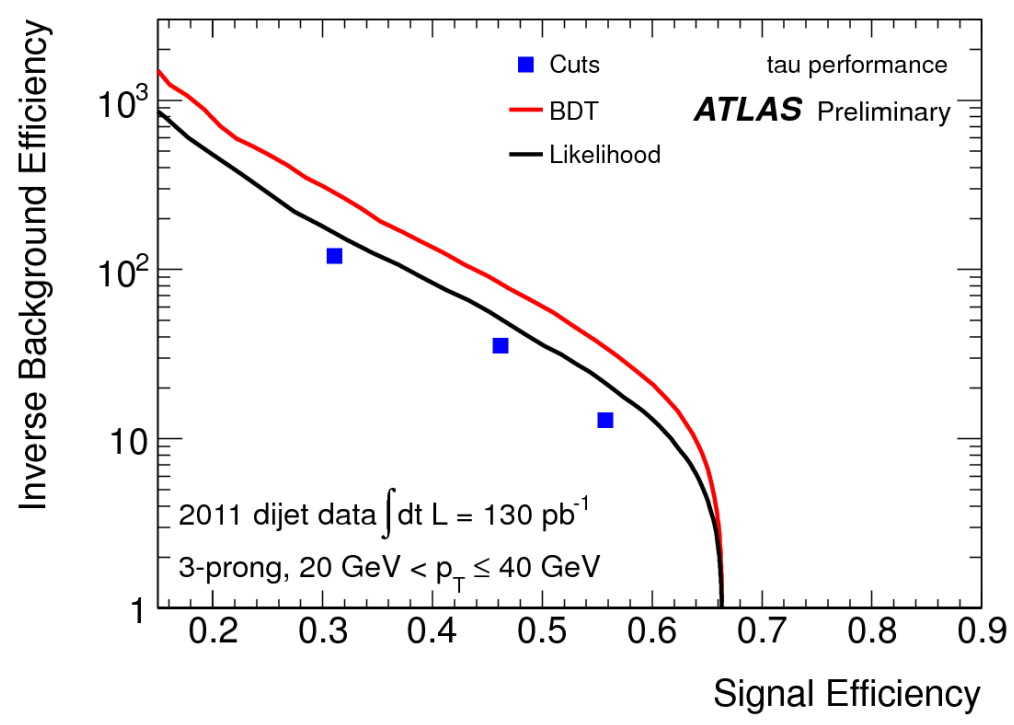

Figure 4: Inverse background efficiency as a function of signal efficiency for each jet discriminant for 3-prong taus in the region $20 \mathrm{GeV}<p_{T}<40 \mathrm{GeV}$ [2]. 


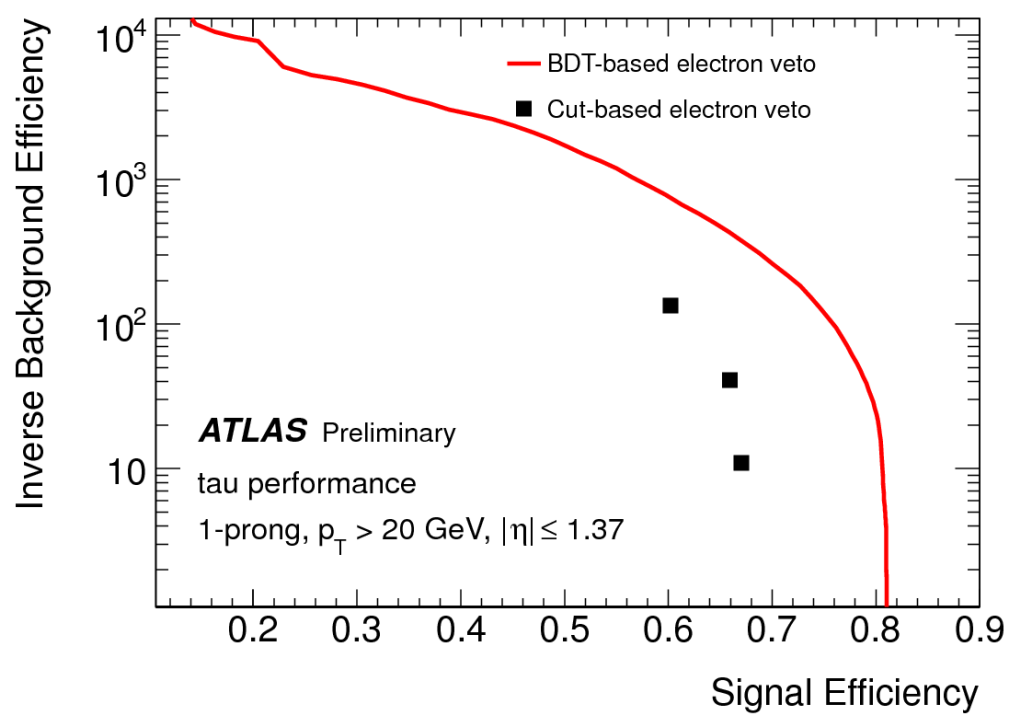

Figure 5: Inverse background efficiency as a function of signal efficiency for each electron discrimination algorithm for 1-prong taus in the central detector region of $|\eta| \leq 1.37$ with $p_{T}<20 \mathrm{GeV}$ [2].

1-prong hadronic tau decays can also be mimicked by electrons. Two algorithms (a cut based and a BDT based) are developed to exploit the differences between electron and hadronic tau decays, most important of which are the number of high threshold transition radiation hits and the shower shape in the calorimeter. The performance of these algorithms in the region $|\eta| \leq 1.37$ is shown in Figure 5.

\section{Identification Efficiency}

The performance of identification algorithms is tested using independent analyses. In the first analysis $Z \rightarrow \tau \tau$ events, selected from 2011 data corresponding to $800 \mathrm{pb}^{-1}$ with criteria very similar to the $Z \rightarrow \tau \tau$ cross-section measurement [3], are used. This analysis makes use of a "tag and probe" approach, where the event is "tagged" using a leptonically decaying tau with the requirement that there is an accompanying hadronic tau decay(the probe). The background is dominated by multi-jet events, which are estimated using a data driven method. All other backgrounds are estimated through simulation. The invariant mass of the visible decay products for events selected is shown in Figure 6 before identification is applied and in Figure 7 after Tight BDT identification. Signal efficiency is estimated by counting events before and after each tau identification is applied. No significant deviation between data and simulation is observed.

Tau identification efficiency is also measured using $W \rightarrow \tau \nu$ events selected from 2011 data corresponding to $1.37 \mathrm{fb}^{-1}$. In this analysis events are tagged by the large missing transverse momentum and the number of taus is estimated by a fit to the distribution of the number of tracks associated to a tau candidate. Three template distributions are considered. One for true tau leptons decaying to hadrons, one for misidentified electrons and one for misidentified QCD jets. Of these the first two are taken from simulation and the last is extracted from data in a control region. The number of tracks distribution for events selected is shown in Figure 8 before identification 


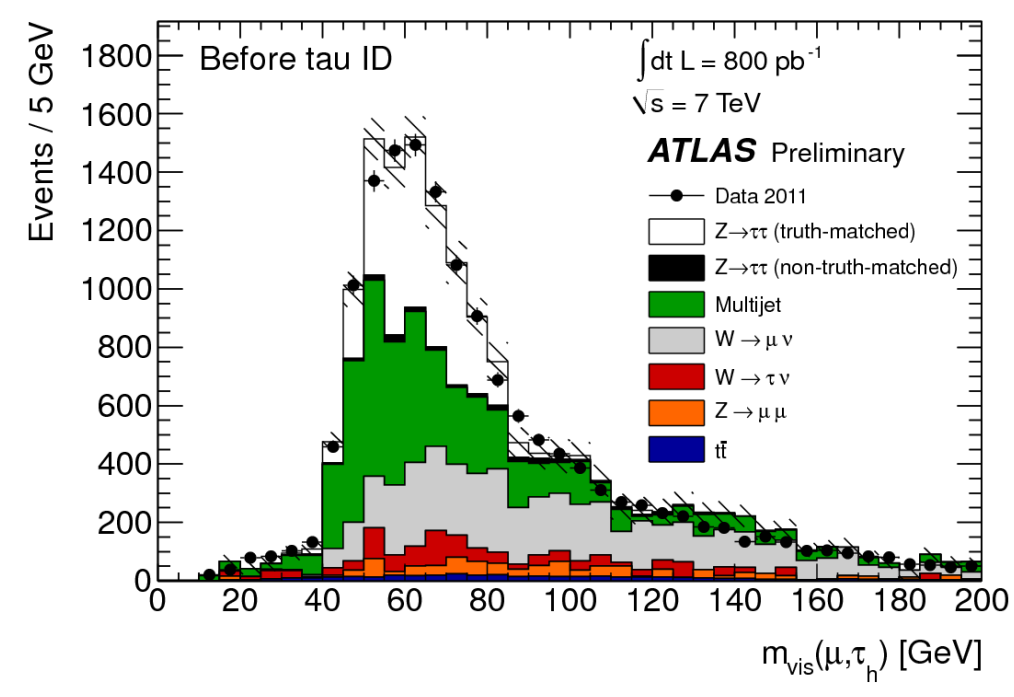

Figure 6: Visible mass distribution after full event selection without any identification requirement on the tau that decays to hadrons [2].

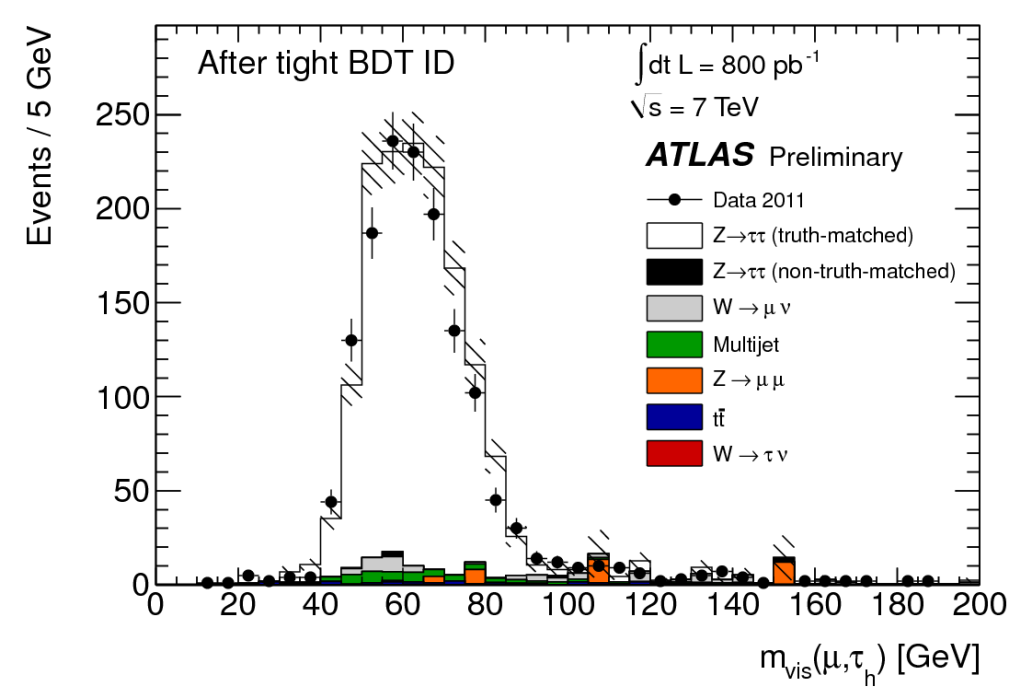

Figure 7: Visible mass distribution after full event selection and requiring the tau that decays to hadrons to pass Tight BDT identification [2].

is applied and in Figure 9 after Tight BDT identification. There is agreement between data and simulation across all identification algorithms to a precision of $\approx 5 \%$ [2].

\section{Energy Calibration}

The energy clusters, which form the reconstructed jets that seed the tau reconstruction, are already calibrated using local hadron calibration (LCW) [4]. An additional correction, derived from simulation, is applied to restore the tau energy to the true value [5]. This correction is applied 


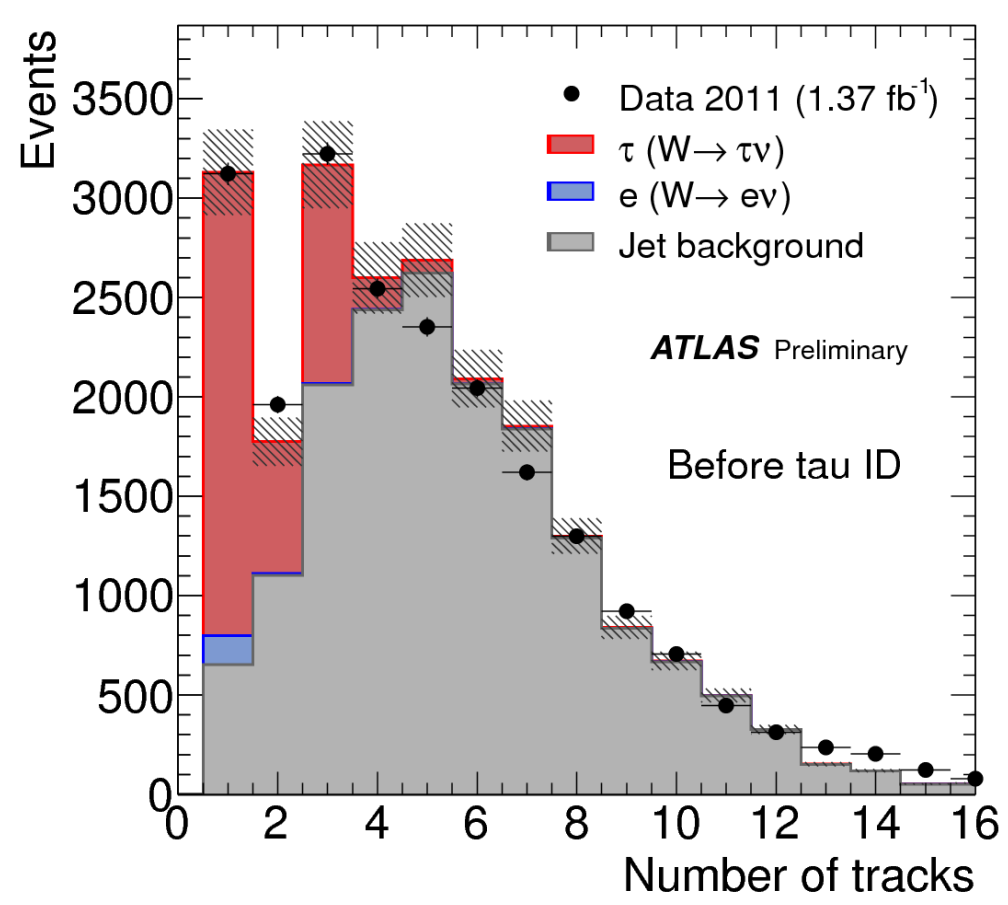

Figure 8: Number of tracks distribution after full event selection without any identification requirement on the tau that decays to hadrons. Each of the three templates are shown [2].

in bins of $\eta, E_{L C W}$ and number of prongs. Figure 10 shows the reconstructed tau energy $\left(\mathrm{E}_{\text {reco }}^{\tau}\right)$ divided by true tau energy as a function of $\mathrm{E}_{\text {reco }}^{\tau}$ for 1-prong decays.

The systematic uncertainty on the tau energy calibration is derived from a combination of simulation and data. Figure 11 shows each contribution to the total tau energy calibration systematic in bins of reconstructed tau momentum for 1-prong decays with $|\eta|<0.3$. The total systematic uncertainty on the calibration is $3 \%$ on average and up to $5 \%$, in the region $0.8<|\eta|<1.6$ for tau transverse momentum $>20 \mathrm{GeV}[5]$.

\section{Summary}

ATLAS has a large number of physics goals which require well reconstructed hadronic decays of tau leptons in the final state. The tau identification algorithms provide sophisticated methods with strong discrimination against backgrounds, with the multivariate techniques performing better in all categories. This performance is measured in data and found to agree with simulation to a precision of $\approx 5 \%$. The energy scale of tau leptons decaying to hadrons is also well understood, with an average uncertainty of $3 \%$ for 1-prong with $|\eta|<0.3$. 


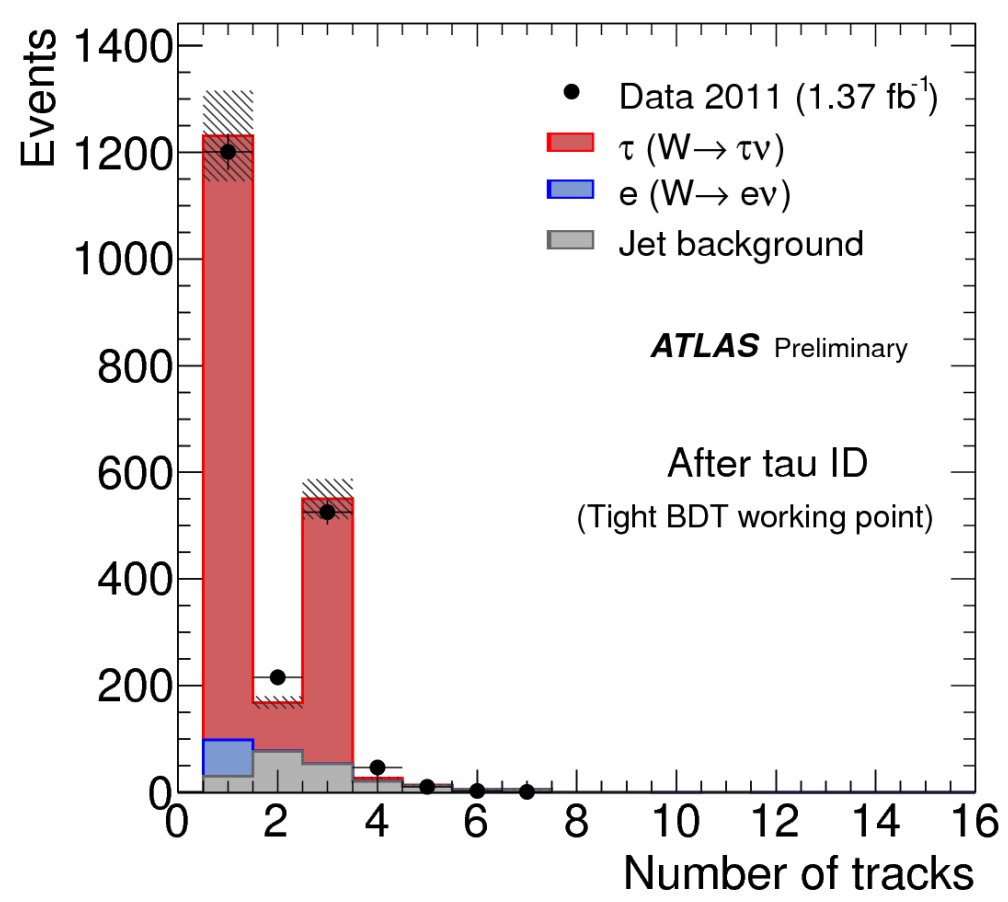

Figure 9: Number of tracks distribution after full event selection and requiring the tau that decays to hadrons to pass Tight BDT identification. Each of the three templates are shown [2].

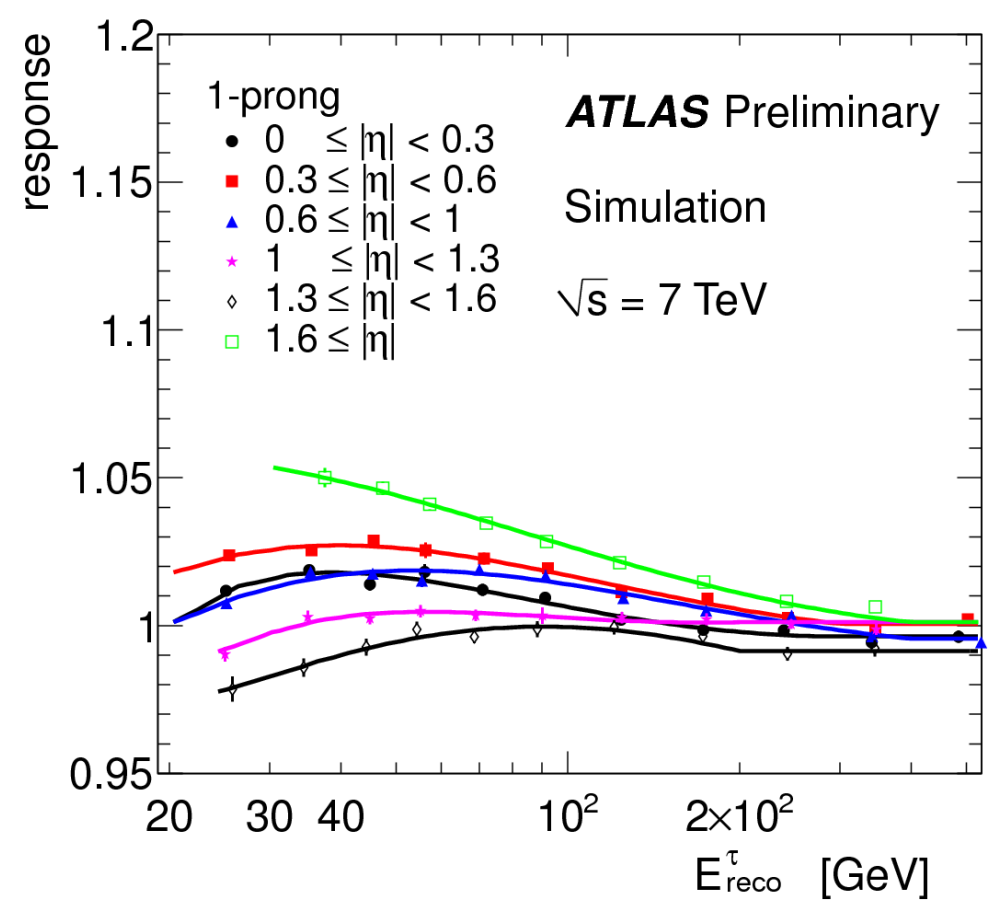

Figure 10: Reconstructed tau energy $\left(\mathrm{E}_{\text {reco }}^{\tau}\right)$ divided by true tau energy as a function of $\mathrm{E}_{\text {reco }}^{\tau}$ for taus with 1 associated track. Uncertainties are purely statistical [5]. 


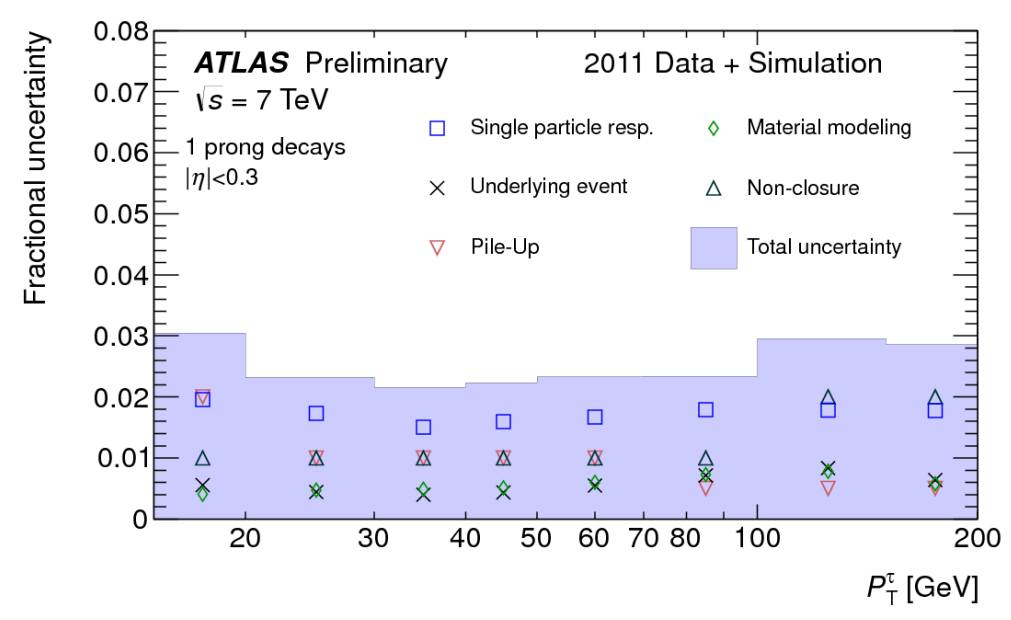

Figure 11: Fractional systematic uncertainty on tau energy scale with each component displayed as a function of reconstructed transverse momentum for 1-prong taus with $|\eta|<0.3$ [5]. 


\section{References}

[1] M. Cacciari, G. P. Salam, and G. Soyez, The anti-kt jet clustering algorithm, JHEP 0804 (2008) 063.

[2] The ATLAS collaboration, Performance of the Reconstruction and Identification of Hadronic Tau Decays with ATLAS, ATLAS-CONF-2011-152, https://cdsweb.cern.ch/record/1398195.

[3] The ATLAS Collaboration, Measurement of the $Z \rightarrow \tau \tau$ cross section with the ATLAS detector, Phys. Rev. D 84, (2011) 112006.

[4] T. Barillari, et al., Local Hadronic Calibration, ATL-LARG-PUB-2009-001-2, https://cdsweb.cern.ch/record/1112035.

[5] The ATLAS collaboration, Determination of the tau energy scale and the associated systematic uncertainty in proton-proton collisions at sqrts $=7 \mathrm{TeV}$ with the ATLAS detector at the LHC in 2011, ATLAS-CONF-2012-054, https://cdsweb.cern.ch/record/1453781. 